\title{
La enseñanza del español como lengua extranjera a alumnos italófonos: J J ramática y sintaxis en la didáctica actual
}

\author{
Anna Sulai Capponi, Università degli Studi di Perugia, Perugia, Italia
}

Resumen: El estudio de la enseñanza de las lenguas extranjeras ha ido interesando a investigadores de varias disciplinas. En el caso del Español L2 para estudiantes italianos ha tenido un desarrollo particular. Consideradas lenguas parecidas no han encontrado el justo enfoque en las primeras gramáticas. Finalmente, con la toma de conciencia del problema que plantea el estudio de las lenguas afines, se han empezado a considerar analogías y diferencias en una óptica contrastiva. La introducción de las nuevas tecnologías en la enseñanza de la lengua extranjera ha creado nuevas problemáticas. En primer lugar tenemos a docentes "inmigrantes digitales" que se enfrentan con discentes "nativos digitales" $y$, en segundo lugar, los cursos de lengua que encontramos actualmente vienen creados con una visión plurilingüistica en donde, de nuevo, no se da a considerar la interferencia existente entre dos lenguas afines, como italiano y español. Analizamos, entonces, como la didáctica actual se pone frente al elemento sintáctico - gramatical, ámbito en donde el estudiante italiano, que quiere perfeccionarse en español, encuentra mayores dificultades.

Palabras Clave: Español L2, Sintaxis, Enseñanza

Abstract: The teaching of foreign languages has aroused a widespread interest among scholars of various subjects. In particular, the teaching of Spanish as L2 has been specifically developed over the years. Old textbooks have traditionally failed to give a suitable grounding by focusing too much on similarities between the two languages. In more recent times, as scholars have become more conscious of the challenges associated with related languages, they have begun comparing and contrasting. Moreover, the advent of new technologies continues to pose new challenges. One such challenge relates to teachers as "digital immigrants" and learners as "digital natives". Furthermore, new language courses are designed with a plurilinguistic approach and do not take into account the linguistic interferences between Spanish and Italian. This article aims to investigate how modern teaching deals with grammar and syntax as major issues for Italian students aiming to improve their Spanish.

Keywords: Spanish L2, Syntax, Teaching

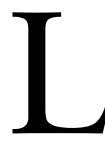

OS AVANCES TECNOLÓGICOS de los últimos años han situado a los docentes frente a nuevas fronteras. El cambio generacional crea desde siempre un pasaje que naturalmente obliga a la reflexión. Lo que se está experimentando en los últimos años, en cualquier caso, tiene un cariz excepcional. Los nuevos medios de comunicación han revolucionado la concepción del tiempo y del espacio. Todo lo que antes necesitaba de ritmo, pausa, movimiento, horarios... ahora ya pertenece al pasado. Antes para encontrar un texto era necesario ir físicamente a un biblioteca, respetando el día y el horario de apertura, esperar el propio turno, pasar horas revisando los ficheros, esperar a que el empleado fuera a recogerlo, rellenar fichas, dejar copia de un documento, respetar la fecha

\footnotetext{
Revista Internacional de Educaci ón y Aprendizaje

Volumen 1, 2013, http://sobrelaeducacion.com/revistas/coleccion/, ISSN 2255-453X

(C) Global Knowledge Academics. Anna Sulai Capponi

Todos los Derechos Reservados. Permisos: soporte@gkacademics.com
}

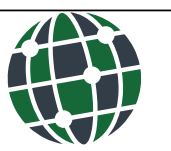


de entrega, tomar el objeto concreto y llevarlo a casa... Todo eso ahora pertenece a una realidad que parece prehistórica. Los docentes también, aunque nacidos en la era pre-Internet, se han conformado al nuevo modo de buscar un texto: sentados en su propio escritorio y, a través de Internet, leyendo cómodamente el texto en cualquier momento, a su gusto. Los estudiantes que hoy el docente encuentra en su aula no han acudido casi nunca a una biblioteca, pues consiguen toda la información directamente en la pantalla del ordenador. Los muchachos de hoy mantienen conversaciones y amistades a través de la Red, en donde encuentran incluso su mejor diversión. Este cambio impresionante tiene sus repercusiones en la enseñanza y el aprendizaje. Por un lado los docentes se esfuerzan por encontrar la justa metodología y los medios adecuados para relacionarse con el alumnado y por otro los estudiantes encuentran dificultades en respetar los tiempos necesarios para el aprendizaje de una lengua extranjera.

El estudio de los idiomas puede emprenderse según varias exigencias y modalidades. Es decir, si necesitamos establecer una relación exclusivamente comunicativa usamos los recursos que nos lleven de manera más rápida a la adquisición de los elementos básicos. Pongamos el ejemplo de un jugador de fútbol que necesita comunicarse en una lengua extranjera porque ha sido contratado por un periodo de tiempo determinado en un país en el que no se habla su lengua materna; es natural que necesite de un conocimiento básico adecuado para la supervivencia, la comunicación oral y la comprensión de los mensajes, de modo que pueda comprender al entrenador y a sus compañeros. Es bastante habitual que el sujeto que tomamos como ejemplo realice un estudio rápido, con la ayuda de aquellos medios fácilmente accesibles en la Red que les permitan alcanzar ágilmente la meta.

Otra realidad es la del estudiante universitario de idiomas. Su meta es la de utilizar sus conocimientos en el mundo laboral, donde la traducción, la interpretación y la comunicación oral y escrita deben tender a la perfección.

La distancia, sin embargo, que se ha ido creando entre docente y discente es un problema que realmente necesita de una meditación profunda y razonada.

"Los estudiantes de hoy no han cambiado de forma paulatina en relación a los del pasado, no han cambiado simplemente su forma de hablar, su ropa, los adornos de su cuerpo, o su estilo, como ha pasado entre generaciones anteriores. Ha ocurrido una gran discontinuidad. Podríamos llamarlo una 'singularidad': un evento que cambia las cosas de manera tan importante que es absolutamente imposible volver atrás. Esta llamada 'singularidad' es la llegada y la rápida diseminación de la tecnología digital en la última década del siglo XX. Los estudiantes universitarios de hoy representan la primera generación que creció con esta nueva tecnología. [...] Es muy serio, porque el problema más importante que enfrenta la educación actual es que nuestros Inmigrantes Digitales, que hablan un lenguaje fuera de época (de la era pre-digital), están luchando para enseñar a una población que habla un lenguaje completamente nuevo. [...] Los Nativos Digitales están acostumbrados a recibir información de una forma realmente rápida. Les gustan los procesos en paralelo y trabajar en múltiples tareas a la vez. Prefieren los gráficos antes que el texto. Prefieren los accesos aleatorios (como el hipervínculo). Funcionan mejor cuando trabajan en redes. Obtienen satisfacción en la gratificación instantánea y las recompensas frecuentes" (Prensky, 2001). 
Partiendo de estas afirmaciones, plantearemos el objeto de nuestro artículo, tratando de ver cómo debe desarrollarse el trabajo de un docente "Inmigrante Digital" frente al alumnado italófono "Nativo Digital" en la enseñanza de la lengua española LE con respecto a la gramática y sintaxis.

Efectivamente los problemas que vamos a abordar son muchos: las dificultades objetivas del docente frente a las nuevas tecnologías; el concepto de espacio-tiempo que no coincide entre las dos generaciones; las dificultades intrínsecas que gramática y sintaxis representan desde siempre, las problemáticas de la enseñanza de lenguas afines...

En los años ' 80 , con el advenimiento de las computadoras, se empezaron a modificar los modos de enseñar y aprender, aunque el uso por parte del docente quedó relegado más bien a lo meramente mecánico debido a la falta de comprensión de la tecnología, pues hay que tener en cuenta que casi nunca había sido instruido en el conocimiento de la informática.

El uso quedó, y queda, mayormente relegado al soporte en los dictados o para la proyección de diapositivas en donde se insertan textos para presentar a un autor o un argumento (Power Point ${ }^{\circledR}$ Microsoft Office), para visualizar estadísticas, diseños, gráficos, etc (Andrada, Parselis, 2009).

Los docentes, en su mayoría, no son contemporáneos de la Era de la Información. En general conciben la computadora como un instrumento y no como lo que realmente es: la más poderosa herramienta de simulación de procesos que el hombre jamás haya inventado (ídem: 9).

La afirmación de Andrada y Parselis (ídem) viene concebida en el contexto de un estudio sobre el e- learning: una nueva frontera en el campo de enseñanza-aprendizaje. El estudiante no participa sólo de oyente sino que está involucrado en los procesos de producción. La figura del docente se trasforma en una especie de mediador/tutor:

“... el e-profesor ha de tener y desarrollar una serie de funciones de carácter pedagógico, de orientación y técnicos, que a su vez exigen una serie de competencias. La intensidad con la que el profesor de formación virtual desempeñe estas funciones y competencias varía dependiendo de las características del programa formativo" (Blázquez Entonado, Alonso Díaz, 2009: 214).

Según estos nuevos modos de considerar la docencia, el profesor debería instruirse para poder afrontar una didáctica revolucionaria, si bien no se habla de los contenidos, de manera que tenemos que pensar que los argumentos serían los mismos. La gramática y la sintaxis, necesarias para un conocimiento profundizado de la lengua, se seguirían enseñando pero de manera innovadora.

Otro cambio sustancial, como ya hemos dicho, se refiere a la relación espacio-temporal. El alumno de hoy vive el espacio-tiempo de forma diferente:

"Tanto en los medios como en la educación el concepto de periodicidad desaparece. Hoy los procesos virtuales son 'al tiempo', no al espacio. En una propuesta educativa multiámbito los alumnos se hacen presentes todo el tiempo y su profesor-tutor también. La discusión del tiempo y espacio es muy profunda ya que se constituye como un eje determinante para la comprensión de este fenómeno. Ya no contamos solamente con la experiencia sensible de los objetos físicos, que son esencialmente al espacio, sino también con la experiencia sobre objetos virtuales a los que accedemos a través de 
procesos, y en muchos casos estos objetos son también procesos en sí mismos. Sólo se verifican procesos en el tiempo. Por lo tanto, toda nuestra experiencia virtual está basada en el tiempo, una experiencia que en un contexto como el de Internet, además, tiene la característica de la construcción de procesos complejos a partir de procesos simples, todos virtuales e intangibles. Por otra parte, la capacidad de replicar esta propuesta en una organización educativa está más relacionada con la capacitación de sus recursos humanos que con los tecnológicos" (ídem: 19).

Pero, si resulta bastante claro que el docente necesita mantenerse al día respecto de los avances incluso para establecer un contacto comunicativo con el discente, menos evidente parece cómo realizar cierto tipo de clase y desarrollar ciertos argumentos como los gramaticales y sintácticos.

A través de los medios informáticos, y aprovechando también el $e$ - learning, podemos afrontar cierto discurso, permitir al estudiante participar en el descubrimiento de los problemas que hay que tratar y acompañarlo en la investigación y en los ejercicios. Pero lo que desde un punto de vista teórico parece de fácil solución se debe conjugar en la realidad práctica.

Hablemos de ejemplos concretos. Una de las primeras lecciones de gramática que el docente de español LE imparte en un aula de estudiantes italoparlantes es sobre los verbos auxiliares. La cuestión presenta varias dimensiones: qué verbos hay que considerar auxiliares (Coronado Hernández, 2008), las diferencias en ámbito contrastivo entre voz activa y pasiva españolitaliano (Núñez Román, 2009), su uso en las construcciones perifrásticas (Luque, 2008), entre otros.

Vamos entonces a ver cómo la Red nos presenta este argumento en una de las páginas que aparece entre las primeras al escribir en un buscador "gramática española online":

- El verbo ser se utiliza como auxiliar en la conjugación pasiva (Ficha 27: La voz pasiva I):

Aquel monarca fue muy apreciado por sus súbditos.

- Comparte las funciones con estar como verbo atributivo (Ficha 26: Usos de ser y estar), confiriendo cada verbo un matiz diferente al atributo:

Esa niña es muy nerviosa.

Esa niña está muy nerviosa.

- El verbo estar se utiliza también como verbo locativo:

Madrid está en el centro de la Península.

Su casa estaba bastante lejos de la nuestra.

- Son muy utilizadas en español las perífrasis compuestas por el verbo estar + gerundio, con valor de continuidad:

Estoy leyendo una novela interesantísima.

Tanto ser como estar participan en muchas otras perífrasis y locuciones (Ficha 43:

Perifrasis de infinitivo I ):

Lo que has hecho por nuestro hijo es muy de agradecer.

Eso está por ver.

Cuando me llamaste estaba para salir.

- El verbo haber sirve como auxiliar en las formas compuestas:

he comido

habíamos salido 
habrás visto

hayáis comprado

haber estado, etc.

El participio de los tiempos compuestos es invariable, y no puede ir separado del auxiliar haber por ninguna otra palabra, de modo que no podemos decir *lo has muy bien expresado sino lo has expresado muy bien.

- Como verbo impersonal se utiliza siempre en tercera persona de singular, teniendo en presente de indicativo una forma particular (hay):

Ha habido un accidente esta mañana.

Había muchísima gente en el concierto.

No hay quien lo entienda.

En estos casos el verbo haber tiene un significado similar al de existir.

- Las perífrasis con el verbo haber tienen significado de obligación, en forma impersonal con haber + que + infinitivo:

Hay que estudiar durante todo el curso.

No hubo que decirle nada, ya lo dedujo él.

y en forma personal con haber + de + infinitivo:

He de estudiar para el examen.

Al llegar hubieron de enseñar sus documentos de identidad.

- También es necesario distinguir entre el uso del verbo haber y el de estar en las indicaciones de lugar:

\begin{tabular}{|l|l|l|}
\hline \multirow{2}{*}{ En esa calle } & $\begin{array}{l}\text { Un supermercado. } \\
\text { algunos coches mal aparcados. } \\
\text { muchas tiendas. } \\
\text { pocos árboles. } \\
\text { tres farmacias. } \\
\text { bares (plural) de copas. } \\
\text { luz }(\boldsymbol{\varnothing}) \text { eléctrica. }\end{array}$ \\
\cline { 2 - 3 } & $\begin{array}{l}\text { EI supermercado. } \\
\text { las farolas encendidas. } \\
\text { ese taller mecánico. } \\
\text { mi casa. } \\
\text { nuestro coche aparcado. }\end{array}$ \\
\hline
\end{tabular}

http://www.auladiez.com/fichas/23_verbos_estar_ser.php

Sigue el ejercicio apropiado:

Complete las siguientes frases con hay, está o están:

\begin{tabular}{|l|l|l|l|l|l|l|l|l|l|}
\hline Pulse para insertar: & á & é & í & ó & ú & ü & $\tilde{n}$ & i & i \\
\hline
\end{tabular}

Inizio modulo 
1. No $\square$ leche en la nevera.

2. Muy cerca de mi apartamento $\square$ una boca de metro.

3. ¿Dónde $\square$ mis llaves?

4. La caldera de la calefacción $\square$ en el sótano.

5. En esa biblioteca $\square$ muchos libros antiguos.

6. Estas sillas $\square$ fuera de su sitio.

7. En la despensa $\square$ dos latas de atún.

8. En el centro $\square$ muchas calles estrechas.

[http://www.auladiez.com/fichas/23_ejercicios.php]

La ficha gramatical no parece adecuada en cuanto no resuelve las dificultades que los verbos auxiliares presentan a un estudiante en general y a un italófono en particular, ni mucho menos las dudas que constantemente plantea el uso de "ser" o "estar":

"A la peculiaridad de los valores diferenciales de ser y estar que caracterizan al español, se suma un destino un tanto incierto en la consideración que de ambos verbos ha realizado la gramática a lo largo del tiempo. La tradición gramatical griega y latina no aportó datos significativos al respecto porque la entidad de sum, de stare o de einai no suponía distinciones semánticas idénticas a las de nuestra lengua. La tradición gramatical española, e incluso diversas corrientes lingüísticas del siglo $\mathrm{XX}$, han mantenido en entredicho la identidad semántica de ser y de estar en el marco de la oración; y no ofrecen aún criterios definitivos para distinguir su uso. Los intentos de encontrar fórmulas de diferenciación resultan todavía insuficientes por la multiplicidad de coordenadas y valores semánticos, pragmáticos y gramaticales que implican oraciones del tipo Juan es bueno, Juan está bueno. [...] La multiplicidad de teorías e interpretaciones y la falta de unanimidad de los criterios que explicarían estos usos, no permiten al docente de ELE responder al alumno con claridad ni seguridad acerca de las maneras de diferenciarlos, ni de las estrategias para el reconocimiento o para la producción de oraciones copulativas con Ser y Estar gramaticalmente aceptables. Cabe preguntarse si, en relación con los usos concretos y paradigmáticos de ser/ estar, a partir de todo este material teórico, de estas reflexiones, podrían derivarse respuestas más menos claras y seguras para estas cuestiones. Lejos de limitarnos a un criterio exclusivo de diferenciación, resulta más útil y eficaz en cuanto al objetivo descriptivo, tomar oportunamente el conjunto de ellos de acuerdo con los casos específicos. [...] Como en otros muchos temas gramaticales, la teoría no debería prescindir del estudio de los contextos y usos reales posibles. Pero tampoco el objetivo didáctico debería desaprovechar la reflexión teórica bien fundamentada que puede dar sentido a explicaciones prácticas y a las pautas de usos comunicativos concretos" (Regueiro Rodríguez, 2008).

Los ejercicios tampoco parecen suficientes para aclarar tantas incertidumbres que le surgen al estudiante abordando esta temática. Perplejidades que el docente debe intentar solucionar incluso con la ayuda de un análisis contrastivo que le permita descifrar los puntos más difíciles para un estudiante italohablante y establecer puntos de contactos en donde los haya.

Según Maria Vittoria Calvi (2004): 
“... la enseñanza de L2 no puede descartar nunca la contrastividad, que corresponde a procedimientos mentales de amplio alcance; este enfoque, además, resulta especialmente aconsejable cuando la distancia entre la L1 y la L2 es poca y es percibida como tal. Por supuesto, hablar de contrastividad no significa volver a los supuestos del viejo AC, sino adoptar un planteamiento más dinámico" (Calvi, 2004).

No se trata de rechazar un camino ya emprendido, pero tampoco de borrar siglos de clase presencial. Como comentábamos al inicio, ya todos hemos tenido la oportunidad de comprobar las ventajas y oportunidades que la tecnología nos ha aportado, de modo que parece fuera de lugar refutar el cambio y no tratar de utilizar estos medios.

Tampoco, sin embargo, el docente puede evitar asumir sus responsabilidades y pensar que su empeño didáctico se solucione con la participación activa del aprendiz. La enseñanza de la gramática y sintaxis representa uno de los retos más significativos, sobre todo una clase italófona. Esto ocurre porque la enseñanza de lenguas afines conlleva dificultades propias en la medida en que la interferencia representa un obstáculo, sobre todo en la tentativa de perfeccionamiento:

“... lo spagnolo offre agli italofoni innegabili vantaggi al primo contatto: l'ordine delle parole nelle frasi è simile, non occorre applicare particolari regole per la forma negativa o per quella interrogativa, a differenza del francese. Quindi le semplici interazioni domanda/risposta non richiedono ingenti sforzi acquisitivi; si osservi ad esempio il parallelismo tra le frasi «¿Cómo estás?» «Bien, gracias, ¿y tú?» e «Come stai?» «Bene, grazie, e tu?», tra «No me gusta el café» e «Non mi piace il caffè», a parte il diverso uso spagnolo dei segni grafici per l'interrogazione. [...] La frustrazione non dipende interamente dal solito carosello di ingannevoli affinità; in buona parte, deriva dal reperimento di vere e proprie difficoltà strutturali dello spagnolo: basta pensare ad alcuni «nodi» come l'uso di ser e estar, la scelta fa indicativo e congiuntivo, l'enorme ricchezza di perifrasi verbali ecc". (Calvi, 1995: 90-91).

Así que un estudiante italohablante, aunque resuelve de manera entusiasta el nivel básico de la comunicación en donde puede aprovechar la transferencia positiva del italiano al español, encuentra tremendas dificultades en ámbito gramatical y sintáctico. Eso sucede porque la distancia estructural es bastante amplia, a diferencia del léxico (en donde se pueden cometer errores por afinidad falsa o verdadera). Resulta, pues, que el estudiante italoparlante procede por correspondencia y el error, en la mayoría de los casos, deriva de su tentativa de recalcar la lengua española sobre una estructura italiana. Lo más difícil para un estudiante italohablante es llegar a enunciar correctamente la frase: "Creo que viene". En efecto la reacción espontánea será la de pronunciar: "Creo que venga", creando un calco de la frase italiana: "Credo che venga", dado que en italiano el verbo "credere" es sinónimo de "supporre", o sea "suponer", creando una situación de incertidumbre y requiriendo, por supuesto, el uso del subjuntivo.

Vamos a otro ejemplo práctico: la alternancia de indicativo/subjuntivo en la oración temporal.

En el glosario de términos lingüísticos usados en el Diccionario Panhispánico de Dudas de la Real Academia Española (2005) aparece la siguiente definición: 
“36. oración temporal. La subordinada que expresa tiempo: Siempre que oigo música, me siento mejor; Me iré cuando haya terminado el trabajo."

Este tipo de oración necesita de una aproximación de contraste con el italiano para su inmediata comprensión, pues "lo habitual" en las oraciones temporales se expresa en italiano también con el indicativo presente: "Ogni volta che ascolto la musica, mi sento meglio". La situación cambia notablemente cuando la oración temporal hace referencia a un tiempo indefinido que en español se expresa con el subjuntivo, mientras que en italiano se da con el futuro indicativo: "Me ne andrò quando avrò terminato il lavoro".

A través de la constrastividad entre estas dos lenguas afines la comprensión resulta mucho más inmediata que a través de una explicación para estudiantes extranjeros en general. Este otro ejemplo, tomado de la página web "El Rincón del Vago", nos muestra lo difícil que es sacar por sí solos las referencias útiles para el conocimiento de este argumento:

\section{PROPOSICIONES SUBORDINADAS ADVERBIALES DE TIEMPO:}

Indican la circunstancia temporal de la oración principal.

Vendré cuando haya terminado de pintar el cuadro.

Van introducidas fundamentalmente por el nexo "cuando" (adverbio relativo), pero pueden aparecer otros nexos o frases según los distintos matices significativos de anterioridad, simultaneidad o posterioridad, reiteración que pueden presentar las proposiciones subordinadas temporales:

Antes de que, antes que, primero que, mientras, mientras que, mientras tanto, entre tanto que, en cuanto, apenas, luego que, así que, después que, desde que, cada vez que, siempre que...

- Llegamos cuando tú salías.

- Antes que te cases, mira lo que haces.

- Mientras seas rico, tendrás muchos amigos.

- En cuanto los vea, les daré un abrazo.

- Después que comamos, hablaremos.

- Siempre que salía a la calle, cogía frío.

- También el valor circunstancial de tiempo puede expresarse:

- En infinitivo precedido de "al" (al salir de clase...)

- En gerundio (corrigiendo los exámenes...)

- En participio (arreglado el coche, nos fuimos) [http:/html.rincondelvago.com/oraciones-subordinadas-adverbiales.html]

Podríamos seguir con muchos ejemplos. Efectivamente todas estas páginas web que encontramos gratuitas en la Red son de una utilidad formidable en cuanto ponen al alcance multitud de textos digitales que en general tienen buena calidad, pues son revisados constantemente por personas competentes. Es como tener una biblioteca en casa. El problema surge en el momento de organizar tanto material. Es donde se necesita la figura del docente, que tiene conocimientos y capacidad de extrapolación. Asimismo, en un aula de italófonos, el profesor será capaz de utilizar la contrastividad cuando sea ventajoso, porque realmente no podemos imaginar que todos estos materiales que encontramos en la Red y que van de las lecciones 
a los ejercicios, a los diccionarios, a los corpora, a los traductores simultáneos, etc., puedan bastar a un alumno universitario. No sólo se necesita un mediador, tutor, interlocutor, sino también un profesor. Aprovechemos el Diccionario de la Real Academia de España (2001) para mostrar la etimología de las palabras:

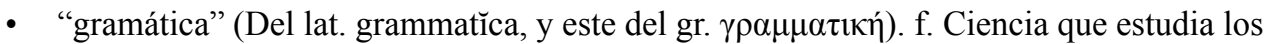
elementos de una lengua y sus combinaciones;

- "sintaxis" (Del lat. syntaxis, y este del gr. $\sigma 0 ́ v \tau \alpha \xi ı \zeta$, de $\sigma v v \tau \alpha ́ \sigma \sigma \varepsilon ı v$, coordinar). f. Gram. Parte de la gramática que enseña a coordinar y unir las palabras para formar las oraciones y expresar conceptos;

- "profesor, ra" (Del lat. professor, -ōris). m. y f. Persona que ejerce o enseña una ciencia o arte;

- "docente" (Del lat. docens, -entis, part. act. de docēre, enseñar). adj. Que enseña. U. t. c. s.;

- “enseñar" (Del lat. vulg. insignāre, señalar). 1. tr. Instruir, doctrinar, amaestrar con reglas o preceptos. 2. tr. Dar advertencia, ejemplo o escarmiento que sirva de experiencia y guía para obrar en lo sucesivo. 3. tr. Indicar, dar señas de algo. 4. tr. Mostrar o exponer algo, para que sea visto y apreciado. 5. tr. Dejar aparecer, dejar ver algo involuntariamente. 6. prnl. Acostumbrarse, habituarse a algo.

- "tutor, ra" (Del lat. tutor, -ōris).1. m. y f. Persona que ejerce la tutela. 2. m. y f. Persona encargada de orientar a los alumnos de un curso o asignatura.

Si nos fijamos en las etimologías precedentes, notamos cómo el tutor orienta mientras que el docente y el profesor enseñan una ciencia; en este caso, se trata de la ciencia que estudia los elementos de la lengua y la coordinación de las palabras para formar oraciones y expresar conceptos.

El estudiante universitario necesita dominar este arte para manejar la lengua en todas sus funciones.

\section{Conclusión}

El profesor universitario de español LE para estudiantes de habla italiana vive hoy nuevas problemáticas que no debe rechazar sino aceptar y usar. En primer lugar debe conformarse a la nueva realidad:

Las instituciones deben convertir la alfabetización tecnológica del profesorado en prioridad y darle formación permanente que les haga más creativos e innovadores en la docencia. La formación abarcará habilidades, actitudes, técnicas didácticas con TIC, elaboración de contenidos on-line, nuevas formas de evaluación con TIC... (Marquès Graells, 2000).

Su objetivo será el de formar a personas competentes capaces de utilizar la lengua extranjera en los más variados ámbitos laborales. La preparación lingüística debe alcanzar los niveles más alto para poder enfrentarse a cualquier dificultad la experiencia laboral les ponga, formando y valorando incluso los aspectos específicos. 
Aunque el discente nacido en la era digital exija tiempos de realización rápidos, el docente debe enseñarle a respetar pautas, ritmos o graduación que acompañan a la comprensión, adquisición y reflexión que el aprendizaje lingüístico comporta.

El docente, además, será el faro en el maremagno de información que el estudiante encuentre navegando en la Red.

La facilidad de encontrar material informático no debe hacer flaquear al docente, que tiene que mantener su empeño didáctico y desarrollar una metodología adecuada para la comprensión y adquisición de los aspectos más complejos, como la gramática y la sintaxis, empleando las nuevas tecnologías sin descuidar la enseñanza.

Con respecto a los estudiantes de habla italiana, debe emplear su competencia para evitar las transferencias negativas y aprovechar las positivas para facilitar la compenetración de los aspectos gramaticales y sintácticos, de los cuales el estudiante italófono entiende la complejidad por similitud pero encuentra dificultad por interferencia.

El profesor hará recapitulación de toda una historia de la enseñanza del español para italianos, resolviendo las lagunas existentes allí donde las primeras gramáticas, muy tardías con respecto a las demás lenguas, no consideraban necesario el estudio gramatical y sintáctico de estos dos idiomas por considerarlos muy parecidos (Calvi, 1995). El riesgo de repetir el error es real, dado que la mayoría de los cursos de lengua online actuales se fundan sobre una visión plurilingüista en la que no se consideran las problemáticas inherentes a las lenguas afines. La didáctica de las lenguas se concibe en una óptica de autoaprendizaje realizada con la ayuda de imagen, sonido y escritura, por lo que ciertos aspectos, como lo abstracto, lo estructural o lo semántico, quedan relegados a un segundo plano y dejan el conocimiento de la lengua a un nivel superficial. El estudiante que pretenda alcanzar una habilidad lingüística que le permita escribir, traducir y hablar correctamente sigue necesitando del aporte del profesor, por su capacidad de instruirlo en aquella ciencia que estudia los elementos de una lengua y sus combinaciones y por su facultad de enseñar a coordinar y unir las palabras para formar las oraciones y expresar conceptos. 


\section{Referencias}

Baralo, M. (2008). "Reflexiones sobre la selección de 'ser' y 'estar' en la interlengua española”. Revista Nebrija de Lingüística Aplicada a la Enseñanza de Lenguas 2/3.

Bautista Pérez, G.; Borges Sáiz F.; Forés i Miravallés A. (2006). Didáctica universitaria en Entornos Virtuales de Enseñanza-Aprendizaje. Madrid, Narcea Ediciones.

Blázquez Entonado F. y L. Alonso Díaz. (2009). "Funciones del profesor de e-learning". Revista de Medios y Educación 34. 205-215. Pixel-Bit.

Calvi, M. V. (1995). Didattica di lingue affini spagnolo e italiano. Guerini Scientifica, Milano.

Luque, R. (2008). "Las perífrasis verbales: un planteamiento contrastivo entre español e italiano". Romanica Cracoviensia 8. 61-69. Jagiellonian University Press.

\section{Webliografía}

Andrada, A. M., y Parselis, M. (2009). E- learning y Educación Superior. Una mirada desde el aprendizaje colaborativo, la interdependencia de saberes y la gestión del conocimiento, Universidad Católica Argentina Instituto de Comunicación Social, Periodismo y Publicidad. [Documento de Internet disponible en http://www.scribd.com/doc/22153056/e-learning-yEducacion-Superior]

Calvi, M. V. (2004). “Aprendizaje de lenguas afines: español e italiano”. Revista Redele 1. [Documento de Internet disponible en http://www.educacion.gob.es/dctm/redele/Material-RedEle/Revista/2004_01/2004_redELE_1_02Calvi.pdf?documentId=0901e72b80e06a6d]

Coronado Hernández, J. 2008. El Verbo Auxiliar. Bibliografía comentada: El verbo auxiliar. EL Colegio de México. [Documento de Internet disponible en http://lef.colmex.mx/Sociolinguistica/Cursos/Bib\%20comentadas/El\%20verbo\%20auxiliar.pdf]

Marquès Graells, P. (2000). "Impacto de las Tic en la Enseñanza Universitaria”. Departamento de Pedagogía aplicada. Facultad de Educación UAB España. [Documento de Internet disponible en http://www.peremarques.net/ticuniv.htm]

Núñez Román, F. (2009). "Diátesis pasiva en italiano y español. Apuntes para un análisis contrastivo" Language Design 11. 79-92. Universidad de Sevilla. [Documento de Internet disponible en http://elies.rediris.es/Language_Design/LD11/LD11-04-Nunnez.pdf]

Prensky, M. (2001) (2010). "Nativos Digitales, Inmigrantes Digital". On the Horizon MCB University Press, 9/5. 1-6. Traducción Vanina Osés, M. y Morelli, P. [Documento de Internet disponible en http://psiytecnologia.files.wordpress.com/2010/02/prensky-nativos-digitales-inmigrantesdigital-traduccion.pdf]

Real Academia Española Diccionario de la lengua española, Vigésima segunda edición (2001) [Documento de Internet disponible en http://lema.rae.es/drae/]

Real Academia Española Diccionario Panhispánico De Dudas, Primera edición (octubre 2005) [Documento de Internet disponible en http://lema.rae.es/dpd/]

Regueiro Rodríguez, M. L. (2008). "Algunas reflexiones sobre ser y estar copulativos en la gramática española”. Revista Nebrija de Lingüistica Aplicada a la Enseñanza de Lenguas 2/3. Universidad Complutense de Madrid. [Documento de Internet disponible en http://www.nebrija.com/revista-linguistica/anteriores/numero1-3/Regueiro_seryestar.pdf] 


\section{Sobre la Autora}

Dra. Anna Sulai Capponi: Dal 1 settembre 2010 collabora come Ricercatrice di Lingua Spagnola presso la Cattedra di Lingua e Letteratura Spagnola della Facoltà di Lettere e Filosofia dell'Università degli Studi di Perugina. Dal novembre 1990 all'agosto del 2010 lavora come Lettrice (convertito in Esperto Linguistico dal 1993) di madre lingua spagnola presso la Cattedra di Lingua e Letteratura Spagnola della Facoltà di Lettere e Filosofia dell'Università degli Studi di Perugia e presso il Centro Linguistico d'Ateneo dell'Università degli Studi di Perugina. Dal 2002 partecipa all'attività didattico/organizzativa prevista per l'indirizzo lingue straniere per i cicli IV, V, VI VII e VIII della Scuola Speciale per Insegnanti di Scuola secondaria (SSIS) dell'Università degli Studi di Perugia, svolgendo le lezioni di "Laboratorio di didattica della lingua e civiltà spagnola". Nel mese di giugno 2001 svolge l'attività di Tutorato Propedeutico per un numero complessivo di 25 ore destinato al potenziamento della conoscenza di base della lingua spagnola presso l'Istituto di Lingua e Letteratura Spagnola della Facoltà di Lettere e Filosofia dell'Università di Perugia. 